\title{
Programm zur Postleitzahlenumstellung
}

Jan Olszerwski,

Maximilian Herberger

Um elektronische Adreßdatenbestände auf das ab dem 1. Juli 1993 geltende, neue, fünfstellige Postleitzahlensystem umzustellen, benötigt man neben einem Umstellprogramm auch Zugriff auf die Leitdateien der Deutschen Bundespost Postdienst. Da diese Leitdateien jedoch zum Teil redundante und zum Teil für die Umstellung nicht benötigte Informationen enthalten, verwendet das für Abonnenten beiliegende Umstellprogramm auf das Nötige reduzierte und in eine optimale Sortierfolge gebrachte Dateien. Diese Dateien haben dBASE-Format und folgende Struktur:

\section{Die Umstelldateien}

\section{UMSDA.DBF}

\begin{tabular}{|r|l|r|}
\hline Feld & Feldname & Länge \\
\hline 1 & PLZWO & 1 \\
\hline 2 & PLZALT & 4 \\
\hline 3 & ORTNAMEA & 40 \\
\hline 4 & ORTZUSA & 30 \\
\hline 5 & NPLZOZ & 1 \\
\hline 6 & NPLZOP & 1 \\
\hline 7 & ZPANSTA & 40 \\
\hline 8 & NZPANSTA & 3 \\
\hline 9 & ALORT & 8 \\
\hline 10 & ORTNAMEN & 40 \\
\hline 11 & PLZZ & 5 \\
\hline 12 & PLZP & 5 \\
\hline & & 179 \\
\hline
\end{tabular}

(Sortierung nach alter Postleitzahl, alle Felder vom Typ „Zeichen”.)

\section{ORTEILDA.DBF}

\begin{tabular}{|r|l|r|}
\hline Feld & Feldname & Länge \\
\hline 1 & ALORT & 8 \\
\hline $2 \cdot$ & ORTNAMF & 40 \\
\hline 3 & OZUS & 30 \\
\hline 4 & ORTTEIL & 40 \\
\hline 5 & NPLZZ & 1 \\
\hline 6 & PLZZ & 5 \\
\hline & & 125 \\
\hline
\end{tabular}

(Sortierung, wie in Originaldatei, nach ALORT - Hauptschlüssel und ORTTEIL. Alle Felder vom Typ „Zeichen”.)

jur-pe 5/93

\section{STRADA.DBF}

\begin{tabular}{|r|l|r|}
\hline Feld & Feldname & Länge \\
\hline 1 & ALORT & 8 \\
\hline 2 & STRLFDNR & 2 \\
\hline 3 & SNAME & 46 \\
\hline 4 & ANZGLEIST & 1 \\
\hline 5 & NPLZZ & 1 \\
\hline 6 & HNRKENN & 1 \\
\hline 7 & HNR1VON & 4 \\
\hline 8 & HNR2VON & 4 \\
\hline 9 & HNR1BIS & 4 \\
\hline 10 & HNR2BIS & 4 \\
\hline 11 & PLZZ & 5 \\
\hline 12 & ORTSTEIL & 40 \\
\hline 13 & PLZWO & 1 \\
\hline 14 & PLZALT & 4 \\
\hline$=$ & & 126 \\
\hline
\end{tabular}

(Sortierung, wie in Originaldatei, nach ALORT - Hauptschlüssel und SNAME. Alle Felder vom Typ „Zeichen”.)

\section{POFADA.DBF}

\begin{tabular}{|r|l|r|}
\hline Feld & Feldname & Länge \\
\hline 1 & ALORT & 8 \\
\hline 2 & NPANST & 3 \\
\hline 3 & PLZWO & 1 \\
\hline 4 & PLZALT & 4 \\
\hline 5 & PFNRVON & 6 \\
\hline 6 & PFNRBIS & 6 \\
\hline 7 & PLZNEUP & 5 \\
\hline & \\
\hline$*$ Gesamt $* *:$ & & 34 \\
\hline
\end{tabular}

(Sortierung, wie in Originaldatei, nach ALORT - Hauptschlüssel -, PFNRVON und PFNRBIS. Alle Felder vom Typ „Zeichen”.)

\section{ORTARDA.DBF}

\begin{tabular}{|r|l|r|}
\hline Feld & Feldname & Länge \\
\hline 1 & PLZWO & 1 \\
\hline 2 & PLZALT & 4 \\
\hline 3 & ORTNAMEA & 40 \\
\hline 4 & ZPANSTA & 40 \\
\hline 5 & NZPANSTA & 3 \\
\hline 6 & ALORT & 8 \\
\hline 7 & ORTNAMEN & 40 \\
\hline 8 & NPLZZ & 1 \\
\hline 9 & PLZZ & 5 \\
\hline 10 & NPLZP & 1 \\
\hline 11 & PLZP & 5 \\
\hline & & 149 \\
\hline
\end{tabular}

(Sortierung, wie in Originaldatei, nach ALORT. Alle Felder vom Typ „Zeichen”.)
jur-pc-Abonnenten können sich diese Dateien mit Hilfe der PLZROM, die dem März/April-Heft beilag, selbst zusammenstellen (Einlesen der Dateien in dBASE, Reduktion auf die benötigten Felder und gegebenenfalls Umsortieren). Wer es einfacher haben will, kann beim Verlag zum Preis von 20,- DM das Update der PLZROM bestellen. Dort sind im Unterverzeichnis IUMSTELL die Dateien in der benötigten Form vorhanden. Das Bestellen dieser CD-ROM hat zugleich den Vorteil, daß man über die aktualisierten Postdateien verfügt. (Die Post hat mittlerweile die seinerzeit ausgelieferten Dateien in zahlreichen Fällen geändert).

Das Umstellprogramm erwartet die Leitdateien in dem Unterverzeichnis, das in der .NPL-Datei angegeben ist. Falls es auf Geschwindigkeit nicht ankommt, kann man hier auch das CD-ROM-Unterverzeichnis direkt angeben.

\section{Installation}

Wenn die Leitdateien verfügbar sind, kopiert man die folgenden Dateien von der Diskette in ein beliebiges Unterverzeichnis:

- CONVERT.EXE

- NPLZ.EXE

- PLZOUT.DBF

- DATEII.CNV

- DATEI2.NPL.

\section{Das Umstellprogramm}

Das Umstellprogramm ist batchorientiert konzipiert, d. h. daß während des Laufs keine Fragen an den Benutzer gestellt werden. Die Umstellung verläuft in zwei Phasen: Zuerst werden die Eingabeadressen in eine „Normalform" konvertiert, danach folgt die Zuordnung der neuen Postleitzahlen.
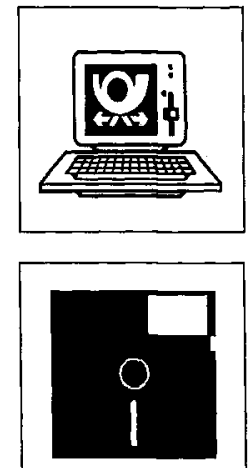

jur-pe 5/93 

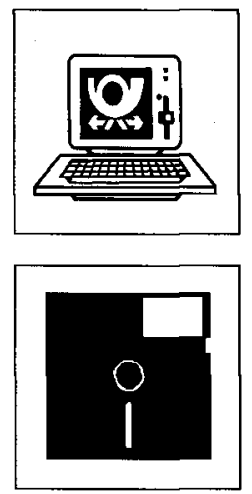

\section{Die Eingabedatei}

Die Eingabedatei mit den eigenen, zu konvertierenden Adressen muß in dBASE-Format vorliegen und nach alten Postleitzahlen sortiert sein. (Gemeint ist die Sortierreihenfolge nach der alten Postleitzahl obne den W/O-Zusatz, also die numerische Reihenfolge.) Informationen zum Aufbau dieser Datei werden in einer Steuerungsdatei an das Programm CONVERT übergeben. Diese Steuerdatei trägt den Namen der Eingabedatei (bis zur Extension) und hat die Erweiterung .CNV. Als Muster ist auf der Diskette die Datei DATEI1.CNV beigegeben. Grundgedanke für diese Datei ist, daß zwischen einer Eingabedatei und einer Ausgabedatei mit möglicherweise unterschiedlichen Feldnamen und unterschiedlicher "logischer" Struktur Zuordnungen hergestellt werden müssen. So enthält beispielsweise die Ausgabedatei ein Feld STRNR für die Straßennummer. Viele Adreßdateien werden die Straßennummer nicht separat führen, sondern sie zusammen mit der Straßenbezeichnung in einem Feld (etwa STRASSE) enthalten. Für diesen Fall bedeutet der Eintrag "STRNR=STRASSE” in der .CNV-Datei, daß die Straßennummer sich im Feld Straße befindet.

Die weiteren Einzelheiten sind in den Kommentarzeilen von DATEI1.CNV erläutert. Durch geeignete Einträge kann man zusätzlich systematischen Besonderheiten oder Fehlern des eigenen Adreßbestandes Rechnung tragen.

\section{Konvertierung}

Das Programm CONVERT wird gestartet mit:

CONVERT EINGABEDATEI AUSGABEDATEI

Die .CNV-Datei wird im Verzeichnis von CONVERT.EXE erwartet.

\section{Zuordnung der neuen PLZ}

Der Programmlauf für die $\mathrm{Zu}-$ ordnung der neuen Postleitzahl wird gestartet mit:

\section{NPLZ EINGABEDATEI}

AUSGABEDATEI

Auch hier wird eine Steuerdatei benötigt (Name der Eingabedatei ohne Extension + .NPL, Musterdatei: DATEI2.NPL mit Kommentaren).

Am Ende des Programmlaufs erscheint eine Statistik, die man mit PrintScreen ausdrucken kann.

\section{Die Nachbearbeitung}

Alle bisherigen Umstelltests haben gezeigt, daß es wohl kaum einen fehlerfreien Adreßbestand gibt. Das Programm verzichtet (abgesehen von den über die .CNV-Datei gegebenen Möglichkeiten) darauf, selbst Fehlerkorrekturen vorzunehmen. Stattdessen enthält die Ausgabedatei im Feld TEST diagnostische Hinweise, die es erlauben, bei den nicht umgestellten Adressen die nötigen Korrekturen vorzunehmen. Man erkennt die nicht umgestellten Dateien an dem Fragezeichen an der letzten Position im Feld "TEST". Das Feld „TEST” enthält fünf Spalten:

1. MSDA.DBF,

2. ORTEILDA.DBF,

3. STRADA.DBF,

4. POFADA.DBF,

5. Diagnose.

\section{Einträge für alle Dateien:}

Leerstelle $=$

Neue Postleitzahl wurde bereits beim vorherigen Suchlauf in anderer Datei gefunden.

$=$ Datei wurde nicht mehr benutzt, weil Information zur Nutzung dieser Datei fehlte (z. B. kein ALORT in UMSDA gefunden.) Datensatz konnte deswegen nicht bearbeitet werden.

Eintrag in Spalte 5:

$+=$ Die Umstellung ist allem Anschein nach gelungen. Es konnte kein Fehler bei der Umstellung beobachtet werden.
? = Es ist bei der Umstellung ein Fehler aufgetreten. Zur Fehlerursache vgl. die folgenden Schlüssel.

\section{Suche in UMSDA}

$1=$ Neue Postleitzahl hier gefunden

$\mathrm{A}=$ Alte Postleitzahl nicht vorhanden

$\mathrm{B}=$ Alte Postleitzahl nicht in zulässiger Form

$\mathrm{C}=$ Keine Ortsangabe in der Eingabedatei

$\mathrm{D}=$ Alte Postleitzahl nicht gefunden

$\mathrm{E}=$ Ort mit alter Postleitzahl nicht gefunden (obwohl Prüfung auf drei Ziffern beschränkt)

$\mathrm{f}=$ ALORT gefunden

\section{Suche in ORTEILDA}

2 = Neue Postleitzahl hier gefunden $\mathrm{g}=$ In der Eingabedatei ist kein Ortsteil enthalten (auch nicht durch Zerlegung eines zusammengesetzten Ortsnamens zu gewinnen)

$\mathrm{h}=$ ALORT nicht gefunden

$\mathrm{i}=$ Ortsteil mit dem ALORT wurde nicht gefunden

j = Es gibt mehrere neue Postleitzahlen für den gefundenen Ortsteil (Programm bricht ab)

Suche in STRADA

$\mathrm{n}=$ Keine Suche in STRADA, da es sich nicht um eine Straße, sondern um ein Postfach handelt

$\mathrm{O}=$ Keine Straßenangabe in der Eingabedatei

$\mathrm{P}=$ ALORT nicht gefunden

$\mathrm{Q}=$ Straße nicht gefunden

$\mathrm{R}=$ Erforderlicher Ortsteil fehlt in der Eingabedatei (es gibt mehrere Straßen gleichen Namens in dem Ort)

$\mathrm{S}=$ Straße in dem betreffenden Ortsteil nicht gefunden

$\mathrm{T}=$ Erforderliche Straßennummer fehlt (es gibt mehrere Postleitzahlen in der Straße)

$\mathrm{U}=$ Bereich, in den die angegebene Straßennummer paßt, nicht gefunden

$3=$ Neue Postleitzahl hier gefunden Suche in POFADA

$\mathrm{x}=$ Keine Postleitzahl in der Eingabedatei vorhanden

$y=$ ALORT nicht gefunden

$\mathrm{z}=$ Erforderliches Zustellpostamt in der Eingabedatei nicht vorhanden

$!=$ Postfach nicht gefunden

$4=$ Neue Postleitzahl hier gefunden

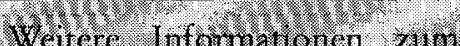
Wentere: ingmonationen: 7 um Bedienung finden sie in der Datei RLADMU IST auf der Diskertenbeliage wir A $\mathrm{Aban}$ nenter. nenen

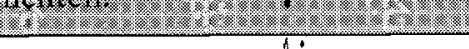

